\title{
The Convergence of Two Algorithms for Compressed Sensing Based Tomography
}

\author{
Xiezhang Li*, Jiehua Zhu \\ Department of Mathematical Sciences, Georgia Southern University, Statesboro, USA \\ Email: *xli@georgiasouthern.edu,jzhu@georgiasouthern.edu
}

Received November 10, 2012; revised December 12, 2012; accepted December 20, 2012

\begin{abstract}
The constrained total variation minimization has been developed successfully for image reconstruction in computed tomography. In this paper, the block component averaging and diagonally-relaxed orthogonal projection methods are proposed to incorporate with the total variation minimization in the compressed sensing framework. The convergence of the algorithms under a certain condition is derived. Examples are given to illustrate their convergence behavior and noise performance.
\end{abstract}

Keywords: Compressed Sensing; Image Reconstruction; Total Variation Minimization; Block Iterative Methods

\section{Introduction}

The reconstruction of an image from the projection data in tomography by algebraic approaches involves solving a linear system

$$
A x=b,
$$

where the coefficient matrix $A \in R^{m \times n}$ is determined by the scanning geometry and directions, vector $b \in R^{m}$ the projection data obtained from computed tomograpgy (CT) scan and the unknown vector $x \in R^{n}$ the image to be reconstructed. It is assumed that system (1) is consistent and underdetermined $(m<n)$. So it has infinitely many solutions. We seek for a solution such that it recovers the original image as good as possible. It is an illposed problem. In general, the dimension of $x$ is very large, thus the conventional direct methods are not appropriate. One of classic iterative algorithms is the algebraic reconstruction technique (ART) given by [1]

$$
x^{k+1}=x^{k}+\lambda_{k} \frac{b_{i}-\left\langle a^{i}, x^{k}\right\rangle}{\left\|a^{i}\right\|_{2}^{2}} a^{i},
$$

where $\lambda_{k}$ is a parameter, $a^{i}$ the $i$ th column of $A^{\mathrm{T}}$, and $\left\langle a^{i}, x^{k}\right\rangle$ the inner product of $a^{i}$ and $x^{k}$. The value of $i$ is cyclically chosen as $1, \cdots, m$. The sequence $\left\{x^{k}\right\}$ converges to a solution of system (1) as long as

$0<\liminf \lambda_{k} \leq \limsup \lambda_{k}<2$ [2]. Cimmino method [3], given by

$$
x^{k+1}=x^{k}+\frac{\lambda_{k}}{m} \sum_{i=1}^{m} \frac{b_{i}-\left\langle a^{i}, x^{k}\right\rangle}{\left\|a^{i}\right\|_{2}^{2}} a^{i},
$$

"Corresponding author. is an iterative projection algorithm suitable for parallel computing. The slow convergence of Cimmino method because of the large value of $m$ was improved by the component averaging (CAV) method [4] given by

$$
x_{j}^{k+1}=x_{j}^{k}+\frac{\lambda_{k}}{s_{j}} \sum_{i=1}^{m} \frac{b_{i}-\left\langle a^{i}, x^{k}\right\rangle}{\left\|a^{i}\right\|_{2}^{2}} a^{i}, j=1, \cdots, n,
$$

where $s_{j}$ is the number of nonzero entries in the $j$ th column of $A$. The CAV method was further generalized as the Diagonally-Relaxed Orthogonal Projection (DROP) method [5] given by

$$
x_{j}^{k+1}=x_{j}^{k}+\frac{\lambda_{k}}{s_{j}} \sum_{i=1}^{m} w_{i} \frac{b_{i}-\left\langle a^{i}, x^{k}\right\rangle}{\left\|a^{i}\right\|^{2}} a_{j}^{i}, j=1, \cdots, n,
$$

where $w_{i}$ 's are positive weights. The block versions of these iterative methods were investigated regarding convergence and computations [5-7]. The theory of compressed sensing [8-10] has recently shown that signals and images that have sparse representations in some orthonormal basis can be reconstructed at high quality from much less data than what the Nyquist sampling theory [11] requires. In many cases in tomography, a medical image can be approximately modeled to be essentially piecewise constant so its gradients are sparse. The image can then be reconstructed via the total variation minimization $[12,13]$. In other words, a two dimensional image $F$ can be reconstructed by solving the following minimization problem,

$$
\min |x|_{T V} \text { or } \min \sum|\mu| \text { s.t } A x=b,
$$

where $|\mu|$ is the magnitude of a gradient and the total 
variation $|f|_{T V}$ of a two dimensional array $f_{i, j}$ is the $l_{1}$ norm of the magnitude of the discrete gradient,

$$
|f|_{T V}=\sum_{i, j} \sqrt{\left(f_{i+1, j}-f_{i, j}\right)^{2}+\left(f_{i, j+1}-f_{i, j}\right)^{2}} \text {. }
$$

Under the condition that the gradients are sparse enough, the solution of the $l_{1}$-norm minimization problem (4) is unique and it gives an exact recovery of the image based on compressed sensing theory $[8,10]$. A block cyclic projection for compressed sensing based tomograph (BCPCS) for solving (4) was proposed [14]. To describe the algorithm the following notations are needed: Suppose that $A$ and $b$ in are partitioned, respecttively, as

$$
A=\left[\begin{array}{c}
A_{1} \\
\vdots \\
A_{p}
\end{array}\right] \text { and } b=\left[\begin{array}{c}
b^{1} \\
\vdots \\
b_{p}
\end{array}\right] \text {, for } 1 \leq p \leq m,
$$

where $A_{j} \in R^{r j \times n}$ and $b_{j} \in R^{r j}, 1 \leq j \leq p$. Assuming that the row indices of $A_{j}$ form a set $B_{j}=\left\{t_{1}^{j}, \cdots, t_{r j}^{j}\right\}$, we have $\mathrm{U}_{j=1}^{p} B_{j}=\{1, \cdots, m\}$ where $B_{i} \cap B_{j}=\varnothing$ for $i \neq j$. We assume that $\beta_{k}>0$ and $\sum_{k} \beta_{k}<\infty$ in the following algorithm:

Algorithm 1. BCPCS

$$
\begin{array}{ll}
\text { 1. for } & k=0,1,2, \cdots \\
\text { 2. for } & j=1 \text { to } p \\
\text { 3.1. for } & i=t_{1}^{j}, \cdots, t_{r j}^{j} \\
\text { 3.2. } & x^{k}=x^{k}+\lambda_{k} \frac{b_{i}-\left\langle a^{i}, x^{k}\right\rangle}{\left\|a^{i}\right\|^{2}} a^{i}
\end{array}
$$

\section{3. end}

4.

$$
x^{k}=x^{k}-\beta_{k} \frac{d}{\|d\|_{\infty}}, \text { where } d=\frac{\partial}{\partial_{x}} \sum|\mu|
$$

5. end

6. $\quad x^{k+1}=x^{k}$

7. end

The convergence of BCPCS with $\lambda_{k}=1$ was shown with an application of the convergence theory of the amalgamented projection method [15].

Theorem 1. [14] The sequence $\left\{x^{k}\right\}$ generated by Algorithm 1 with $\lambda_{k}=1$ converges and its limit is a solution of $A_{x}=b$.

In this paper, the block component averaging and diagonally-relaxed orthogonal projection methods are proposed to incorporate with the total variation minimization in the compressed sensing framework. The convergence of the algorithms, under a certain condition for example in the strip-based projection model [16], is derived. Examples are given to illustrate their convergence behavior and noise performance.

\section{BCAVCS Algorithm and Its Convergence}

The convergence of the CAV and block CAV methods was proved [4,5]. Several notations and concepts in [4] are adopted in this paper for literature consistency. For each $i \in\{1, \cdots, m\}$, denote by

$$
H_{i}=\left\{x \in R^{n} \mid\left\langle a^{i}, x\right\rangle=b_{i}\right\}
$$

a hyperplane in $R^{n}$ and by $G_{i}=\operatorname{diag}\left\{g_{i 1}, \cdots, g_{i n}\right\}$ a nonnegative diagonal matrix, where $g_{i j}=1 / s_{j}$ if $a_{j}^{i} \neq 0$, otherwise $g_{i j}=0, j=1, \cdots, n$. Then $\sum_{i=1}^{m} G_{i}=I$. The set $\left\{G_{i}\right\}_{i=1}^{m}$ is called sparsity pattern oriented (SPO) w.r.t. A. The generalized oblique projection of a vector $z \in R^{n}$ onto $H_{i}$ w.r.t. $G_{i}$, denoted as $P_{H_{i}}^{G_{i}}(z)$, is defined for all $j=1, \cdots, n$, by

$$
\left(P_{H_{i}}^{G_{i}}(z)\right)_{j}= \begin{cases}z_{j}+\frac{b_{i}-\left\langle a^{i}, z\right\rangle}{\sum_{l=1, g_{i l} \neq 0}^{n} \frac{a_{l}^{i}}{g_{i l}}} \cdot \frac{a_{j}^{i}}{g_{i l}} \text { if } g_{i j} \neq 0 \\ z_{j} \quad \text { if } g_{i j}=0,\end{cases}
$$

a seminorm in $R^{n}$ corresponding to any nonnegative diagonal matrix $G$ is defined by $|z|_{G}=\langle z, G z\rangle^{1 / 2}, z \in R^{n}$. It is known that

$$
P_{H_{i}}^{G_{i}}(z)=\operatorname{argmin}\left\{|x-z|_{G_{i}}: x \in H_{i}\right\},
$$

which explains the meaning of the generalized oblique projection. For simplicity, we denote $P_{H_{i}}^{G_{i}}(z)$ by $Q_{i}(z)$ for any $z \in R^{n}$.

Moreover, with the generalized inverse of $G_{i}$,

$$
G_{i}^{\dagger}=\operatorname{diag}\left\{g_{i 1}^{\dagger}, \cdots, g_{\text {in }}^{\dagger}\right\}
$$

where for a real number $\alpha, \alpha^{\dagger}=\alpha^{-1}$ for $\alpha \neq 0$, otherwise $\alpha^{\dagger}=0$, we rewrite (6) as

$$
Q_{i}(z)=P_{H_{i}}^{G_{i}}(z)=z+\frac{b_{i}-\left\langle a^{i}, z\right\rangle}{\left\langle a^{i}, G_{i}^{\dagger} a^{i}\right\rangle} G_{i}^{\dagger} a^{i} .
$$

With above notations, the CAV iteration (2) can be expressed as

$$
x^{k+1}=x^{k}+\lambda_{k} \sum_{i=1}^{m} G_{i}\left(Q_{i}\left(x^{k}\right)-x^{k}\right) .
$$

Algorithm 1 can be modified as a block CAV method for compressed sensing based tomography algorithm (BCAVCS) for image reconstruction described as follows:

$$
\begin{aligned}
& \text { Algorithm 2. BCAVCS } \\
& \text { 1. for } \quad k=0,1,2, \cdots \\
& \text { 2. for } \quad j=1 \text { to } p \\
& \text { 3. } \quad x^{k}=x^{k}+\lambda_{k} \sum_{i \in B_{j}} G_{i}\left(Q_{i}\left(x^{k}\right)-x^{k}\right) \\
& \text { 4. } \quad x^{k}=x^{k}-\beta_{k} \frac{d}{\|d\|_{\infty}}, \text { where } d=\frac{\partial}{\partial x} \sum|\mu|
\end{aligned}
$$

5. end

6. $x^{k+1}=x^{k}$ 
7. end

In order to derive the convergence of Algorithm 2 under a certain condition, we introduce an operator $T_{i}: R^{n}$ $\rightarrow R^{n}$, for $i=1, \cdots, m$, by

$$
T_{i}(z)=z+\lambda_{k} G_{i}\left(Q_{i}-I\right)(z),
$$

where $I$ is the identity operator. For an index vector $t=$ $\left(t_{1}, \cdots, t_{r}\right)$ in $\{1, \cdots, m\}$, we denote $T[t]$ as a composition of operators $T_{t 1}, \cdots, T_{t r}$ by

$$
T[t]=T_{t_{r}} \circ \cdots \circ T_{t_{1}} .
$$

We have the following property for the operator $T[t]$. Lemma 2. Let $\left\{G_{i}\right\}_{i=1}^{m}$ be SPO w.r.t. $A \in R^{m \times n}$ and let $t=\left(t_{1}, \cdots, t_{r}\right)$ be an index vector in $\{1, \cdots, m\}$ such that $G_{k} G_{l}=O$ for any distinct coordinates $k, l$ in $t$. Then for $z \in R^{n}$,

$$
(T[t])(z)=z+\sum_{i \in\left\{t_{1}, \cdots, t_{r}\right\}}\left(T_{i}(z)-I\right)(z) .
$$

Proof. Let $w=T_{h}(z)=\lambda_{k} G_{h}\left(Q_{h}-I\right)(z)+z$. It follows from $G_{l} G_{h}=O$ that

$$
G_{l} w=G_{l} z \text { and }\left\langle a^{l}, w\right\rangle=\left\langle a^{l}, z\right\rangle .
$$

Consequently, we have

$$
G_{l} Q_{l}(w)=G_{l}\left(w+\frac{b_{l}-\left\langle a^{l}, w\right\rangle}{\left|a^{l}\right|_{G_{l}^{\dagger}}^{2}} a^{l}\right)=G_{l} Q_{l}(z) .
$$

Therefore,

$$
\begin{aligned}
& \left(T_{l} \circ T_{h}\right)(z) \\
& =T_{l}(w)=w-\lambda_{k} G_{l} w+\lambda_{k} G_{l} Q_{l}(w) \\
& =z+\lambda_{k} G_{h}\left(Q_{h}-I\right)(z)-G_{l} z+\lambda_{k} G_{l} Q_{l}(z) \\
& =z+\left(T_{l}(z)-I\right)(z)+\left(T_{h}(z)-I\right)(z) .
\end{aligned}
$$

It follows that

$$
\begin{aligned}
(T[t])(z) & =\left(T_{t_{r}} \circ \cdots \circ T_{t_{1}}\right)(z)=T_{t_{r}}\left(\cdots\left(T_{t_{1}} z\right)\right) \\
& =z+\sum_{i \in\left\{t_{1}, \cdots, t_{r}\right\}}\left(T_{i}(z)-I\right)(z) .
\end{aligned}
$$

We complete the proof of the lemma.

The convergence of BCAVCS in a certain case will be shown below using the concepts of SPO matrices and generalized oblique projection. Suppose that $A x=b$ is generated by the strip-based projection model in discrete tomography or CT $[16,17]$. The index vector

$$
t^{j}=\left(t_{1}^{j}, \cdots, t_{r_{j}}^{j}\right),
$$

is associated with the $j$ th subsystem $A_{j} x=b^{j}$ of (5) determined by one projection direction. In each $0-1$ submatrix $A_{j}$, there is exactly one 1 in each column, i.e., $s_{j}=1$, Therefore all rows of $A_{j}$ are orthogonal. So $G_{k} G_{l}=0$ for distinct coordinates $k$ and $l$ in $t^{j}$. In this case we will show the following.
Theorem 3. If each column of every block $A_{j}, 1 \leq j \leq p$, in (5) has exact one 1 then the sequence $\left\{x^{k}\right\}$ generated by Algorithm 2 with $\lambda_{k}=1$ converges and its limit is a solution of (1).

Proof. Recall that the inner loop of BCPCS for the $j$ th block of $A$ can be represented by an operator $P\left[t^{j}\right]$ defined by

$$
P\left[t^{j}\right] x^{k}=\left(P_{t_{r_{j}^{j}}} \circ \cdots \circ P_{t_{1}^{j}}\right) x^{k},
$$

where

$$
P_{i} x^{k}=x^{k}+\lambda_{k} \frac{b_{i}-\left\langle a^{i}, x^{k}\right\rangle}{\left\|a^{i}\right\|_{2}^{2}} a^{i},
$$

then

$$
P_{i}=I+\lambda_{k} G_{i}\left(Q_{i}-I\right)=T_{i},
$$

for all

$$
i \in\left\{t_{1}^{j}, \cdots, t_{r_{j}}^{j}\right\},
$$

and

$$
\begin{aligned}
& T\left[t^{j}\right]\left(x^{k}\right)=\left(T_{t_{r_{j}^{j}}^{j}} \circ \cdots \circ T_{t_{1}^{j}}\right)\left(x^{k}\right) \\
& =\left(P_{t_{r_{j}^{j}}} \circ \cdots \circ P_{t_{1}^{j}}\right)\left(x^{k}\right)=P\left[t^{j}\right]\left(x^{k}\right) .
\end{aligned}
$$

It follows from (9) that the iteration scheme of the inner loop of BCAVCS for the $j$ th block can be expressed as

$$
\begin{aligned}
& x^{k} \leftarrow x^{k}+\lambda_{k} \sum_{i \in B_{j}} G_{i}\left(Q_{i}-I\right) x^{k} \\
& =x^{k}+\lambda_{k} \sum_{i \in B_{j}}\left(T_{i}-I\right) x^{k} \\
& =T\left[t^{j}\right]\left(x^{k}\right)=P\left[t^{j}\right] x^{k} .
\end{aligned}
$$

It means that for the $j$ th block of the matrix $A$ the BCAVCS method is equivalent to BCPCS algorithm. The convergence of BCAVCS as $\lambda_{k}=1$ follows from the convergence of BCPCS algorithm as $\lambda_{k}=1$ by Theorem 1 .

It is remarked that the rate of convergence of BCAVCS in general is close to that of BCPCS in sequential computing. Hence, the convergence of BCAVCS algorithm with parallel computing will be significantly faster than BCPCS algorithm.

\section{BDROPCS Algorithm and Its Convergence}

The convergence of the DROP and block DROP methods was proved [5]. In this section, we propose a block DROP algorithm modified for compressed sensing based tomography (BDROPCS) and show its convergence in a 
certain case. We denote by $s j$ the number of nonzero entries in each column within the $j$ th block and define a DROP operator $\tilde{P}\left[t^{j}\right]$ corresponding to the $j$ th block as

$$
\tilde{P}\left[t^{j}\right] x=x+\frac{\lambda_{k}}{s_{j}} \sum_{i \in B_{j}} w_{i} \frac{b_{i}-\left\langle a^{i}, x\right\rangle}{\left\|a^{i}\right\|^{2}} a^{i} .
$$

Algorithm 3. BDROPCS

1. for $k=0,1,2, \cdots$

2. for $j=1: p$

3. $x^{k}=\tilde{P}\left[t^{j}\right] x^{k}$ with a block DROP

4. $\quad x^{k}=x^{k}-\beta_{k} \frac{d}{\|d\|_{\infty}}$ with $d=\frac{\partial}{\partial x} \sum|\mu|$

5. end

6. $x^{k+1}=x^{k}$

7. end

We first show that in a certain case the DROP is identical to the ART in the following

Theorem 4. Let $A \in R^{m \times n}$ have exactly the same number $s$ of nonzero entries in each column and let rows of $A$ be orthogonal. Then $x^{k+1}$ generated by the DROP in (3) is the same as a vector generated by Algorithm ART with a constant parameter $\frac{\lambda_{k}}{s}$ and weights $w_{i}$.

Proof. Under the assumption, the DROP method can be expressed as

$$
x^{k+1}=x^{k}+\frac{\lambda_{k}}{s} \sum_{i=1}^{m} w_{i} \frac{b_{i}-\left\langle a^{i}, x^{k}\right\rangle}{\left\|a^{i}\right\|^{2}} a^{i} .
$$

For convenience, we rewrite Algorithm ART with a constant parameter $\frac{\lambda_{k}}{s}$ and weights $w_{i}$ for one cycle which yields $x^{k+1}$ from $x^{k}$ as follows:

$$
x^{[1]}=x^{k},
$$

for $i=1$ to $m$

$$
x^{[i+1]}=x^{[i]}+\frac{\lambda_{k}}{s}\left(w_{i} \frac{b_{i}-\left\langle a^{i}, x^{[i]}\right\rangle}{\left\|a^{i}\right\|^{2}} a^{i}\right),
$$

end

$$
x^{k+1}=x^{[m+1]} .
$$

By the assumption on $A,\left\langle a^{i}, a^{l}\right\rangle=0$ for $\mathrm{i} \neq 1$. It follows that

$$
\left\langle a^{i+1}, a^{[i+1]}\right\rangle=\left\langle a^{i+1}, x^{[i]}\right\rangle .
$$

It is easy to see by induction

$$
\left\langle a^{i}, x^{[i]}\right\rangle=\left\langle a^{i}, x^{[1]}\right\rangle=\left\langle a^{i}, x^{k}\right\rangle, i=1, \cdots, m \text {. }
$$

Then (12) can be rewritten as

$$
x^{[i+1]}=x^{[i]}+\frac{\lambda_{k}}{s}\left(w_{i} \frac{b_{i}-\left\langle a^{i}, x^{k}\right\rangle}{\left\|a^{i}\right\|^{2}} a^{i}\right),
$$

from which, the vector $x^{k+1}$ is given by

$$
x^{k+1}=x^{k}+\frac{\lambda_{k}}{s} \sum_{i=1}^{m} w_{i} \frac{b_{i}-\left\langle a^{i}, x^{k}\right\rangle}{\left\|a^{i}\right\|^{2}} a^{i},
$$

which is the same as the vector yielded by the DROP method in one cycle (11).

We now have the convergence of Algorithm 3 in the case where $A x=b$ is generated by the strip-based projecttion model in DT or CT $[16,17]$, which is a direct result of Theorems 1 and 5 .

Theorem 5. If each column of every block $A_{j}, 1 \leq j \leq p$, in (5) has exact one 1 then $\left\{x^{k}\right\}$ generated by Algorithm 3 with $\lambda_{k}=w_{i}=1$ converges and its limit is a solution of $A x=b$.

It is remarked that BCAVCS is a special case of BDROPCS when $w_{i}=1$. Therefore, Theorem 5 can be considered as an alternative proof of Theorem 3 without using the generalized oblique projection. However, it is believed that the concept of generalized oblique projecttion and the idea in the proof of Theorem 3 will be important for our further investigation of the convergence of BCAVCS in a general case.

\section{Numerical Simulations}

In order to test the performance of our algorithms in reconstructing images, we implemented algorithms BCAVCS and BDROPCS in Matlab. The performance of the algorithms was compared with some other algorithms. Algorithm BCAV is a non-CS iterative method formed by deleting line 4 in Algorithm BCAVCS so that no perturbation for total variation minimization is performed. Algorithm CAVCS is a nonblock CAV CS-based iterative method obtained by exchanging lines 4 and 5 in Algorithm BCAVCS so that a perturbation for total variation minimization is performed only after all the blocks are done. Note that in our case BDROPCS is same as BCAVCS. We also tested the sensitivity of the algorithms to additional Gaussian noise. We perform the reconstruction of the $256 \times 256$ Shepp-Logan phantom from a set of 20 reasonably distributed projection directions of rational slopes $[16,17]$. The system $A x=b$ generated by the strip-based projection model, where $A \in$ $R^{20918 \times 65536}$ is highly underdetermined. Moreover, $A$ is a sparse 0-1 matrix and there is one and only one 1 in each column within each of total 20 blocks [17].

The experiment data with the algorithms are summarized in Table 1. We set maximum 500 iterations in the 
test and algorithms will terminate if the relative error reaches 0.001 . The relative error

$$
\frac{\|f-G\|_{2}}{\|f\|_{2}} \text { and } \mathrm{MSE}=\frac{\sum_{m} \sum_{n}(f(m, n)-G(m, n))^{2}}{M \times N}
$$

for a reconstructed image $G$ are used to measure the error. The re-constructed images and relative errors by different algorithms are shown in Figure 1. Our experiment results indicate that algorithms BCAVCS and BDROPCS converge. Algorithm BCAVCS is demonstrated to be much better than BCAV and CAVCS and to have the same converges rate as BDROP. It is remarked that BCAVCS/BDROPCS algorithm is appropriate for parallel computing.

We also tested the algorithms with a real CT image of cardiac [18] of size $256 \times 256$ from projections in 32 directions. The CT image was preprocessed to have a certain gradient sparsity. The reconstructed images and corresponding convergence curves are shown in Figure 2. The experiment indicates that the BCAVCS and BDROPCS are superior to non-CS methods too.

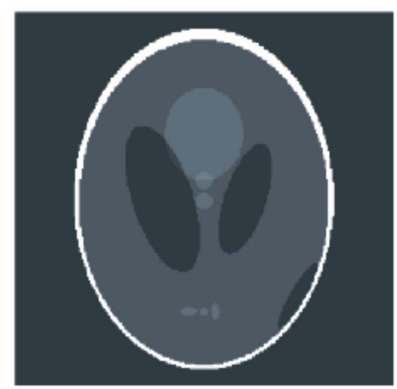

(a)

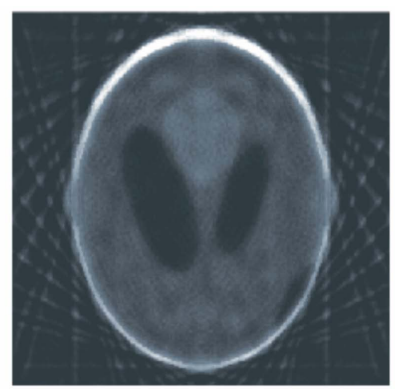

(b)

To evaluate the noise characteristics of two new algorithms, we added Gaussian noise with mean 0 and standard deviation 0.05 to synthetic projection data from the Shepp-Logan phantom. The reconstructed images and convergence curves after 250 itertaions are given in Figure 3. The noise level was evaluated by the mean-square difference between reconstruction with and without added noise. The noise measures with the algorithms BCAV, CAVCS, and BCAVCS/BDROPCS are 0.0012, 0.0029, and 0.0023 , respectively. The results indicate that the four algorithms have simliar sensitivity to data noise.

Table 1. Experiment data of Shepp-Logan phantom.

\begin{tabular}{ccccc}
\hline & $\begin{array}{c}\text { Iteration } \\
\text { Number }\end{array}$ & Time (s) & Error & MSE \\
\hline BCAV & 500 & 55 & 0.458 & 0.013 \\
CAVCS & 500 & 57 & 0.075 & 0.073 \\
BCAVCS & 404 & 75 & 0.001 & 0.000 \\
\hline
\end{tabular}

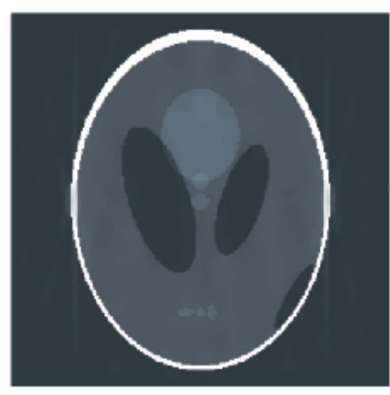

(c)

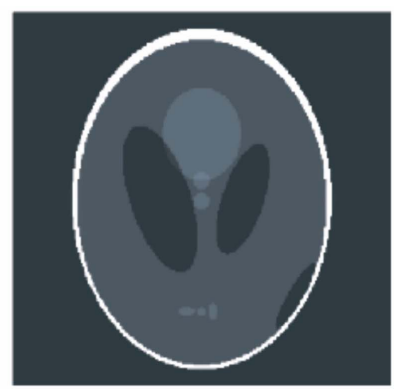

(d)

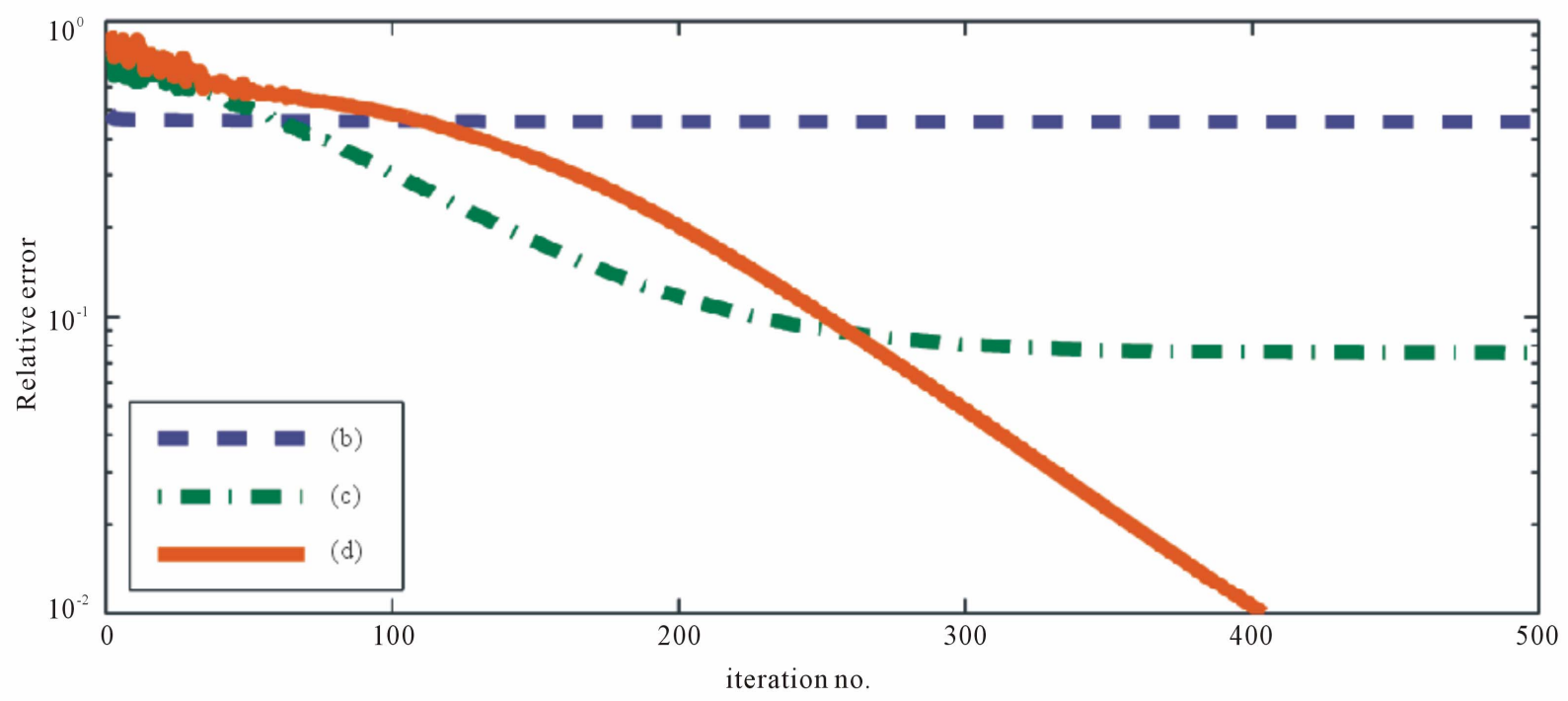

Figure 1. Reconstruction of Shepp-Logan phantom with different algorithms; (a) Shepp-Logan phantom; (b) Reconstruction by BCAV; (c) Reconstruction by CAVCS; (d) Reconstruction by BCAVCS/BDROPCS. Bottom: Convergence curves of algorithms. 


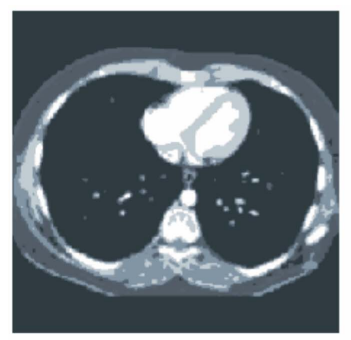

(a)

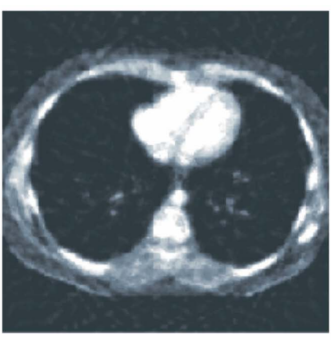

(b)

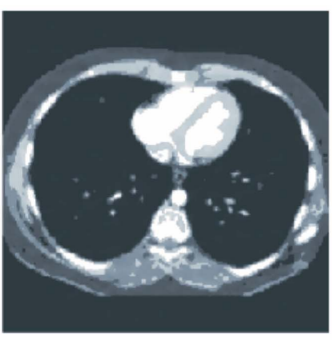

(c)

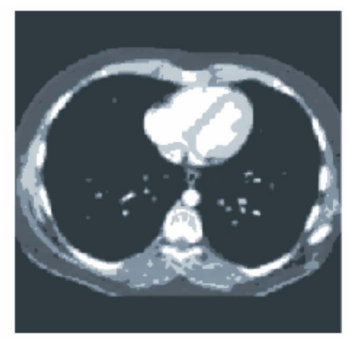

(d)

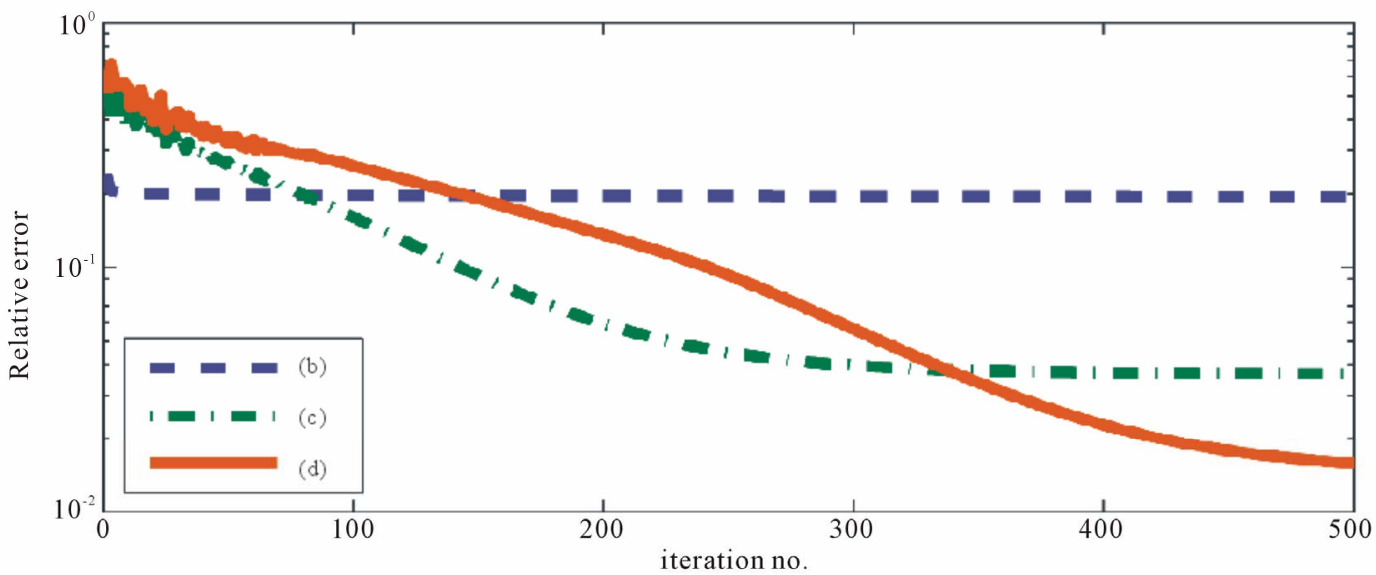

Figure 2. Reconstruction of a cardiac CT image with different algorithms; (a) Cardiac phantom; (b) Reconstruction by BCAV; (c) Reconstruction by CAVCS; (d) Reconstruction by BCAVCS/BDROPCS. Bottom: Convergence curves of algorithms.

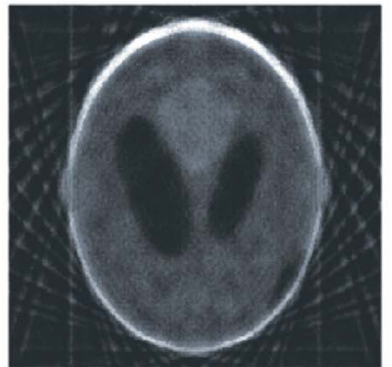

(a)

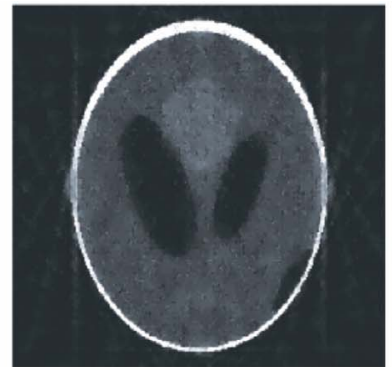

(b)

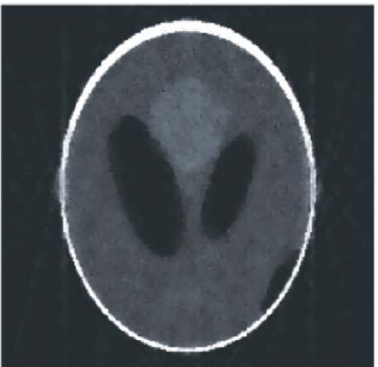

(c)

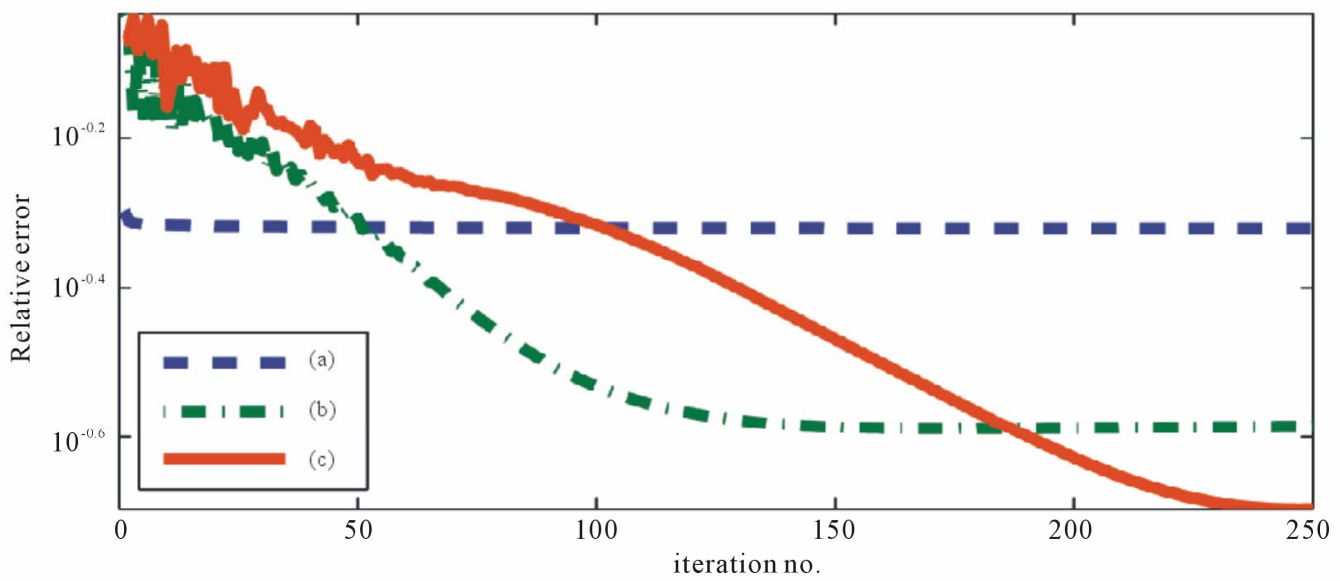

Figure 3. Reconstruction of Shepp-Logan phantom with noise; (a) Reconstruction by BCAV; (b) Reconstruction by CAVCS; (c) Reconstruction by BCAVCS/BDROPCS. Bottom: Convergence curves of algorithms. 


\section{Conclusions}

The total variation minimization is a powerful method to reconstruct piecewise constant medical images based on the compressed sensing theory. We consider the block component averaging and diagonally-relaxed orthogonal projection methods, in the case of the parameter $\lambda_{k}=1$, with the total variation in the compressed sensing framework. Their convergence is derived in the striped-based projection model.

The experiments indicate that the proposed algorithms BCAVCS and BDROPCS converge faster than algorithms without using block iterations or CS framework. Moreover, algorithms BCAVCS and BDROPCS recover more details of images. The convergence of algorithms BCAVCS and BDROPCS in the general case of $\lambda_{k} \neq 1$ will be further studied.

\section{Acknowledgements}

This study was supported by a Faculty Research Award from Georgia Southern University.

\section{REFERENCES}

[1] A. C. Kak and M. Slaney, "Principles of Computerized Tomographic Imaging," Society of Industrial and Applied Mathematics, Philadelphia, 2001. doi: $10.1137 / 1.9780898719277$

[2] P. B. Eggermont, G. T. Herman and A. Lent, "Iterative Algorithm for Larger Partitioned Linear Systems, with Applications to Image Reconstruction," Linear Algebra and Its Applications, Vol. 40, 1981, pp. 37-67. doi:10.1016/0024-3795(81)90139-7

[3] G. Cimmino, "Calcolo Approssimato Per le Soluzioni dei Sistemi di Equazioni Lineari," La Ricerca Scientifica, Series II, Vol. 9, 1938, pp. 326-333.

[4] Y. Censor, D. Gordan and R. Gordan, "Component Aveging: An Efficient Iterative Parallel Algorithm for Large and Sparse Unstructured Problems," Parallel Computing, Vol. 27, No. 6, 2001, pp. 777-808. doi:10.1016/S0167-8191(00)00100-9

[5] Y. Censor, T. Elfving, G. T. Herman and T. Nikazad. "On Diagonally-Relaxed Orthogonal Projection Methods," SIAM Journal on Scientific Computing, Vol. 30, No. 1, 2008, pp. 473-504. doi:10.1137/050639399

[6] R. Aharoni and Y. Censor, "Block-Iterative Projection Methods for Parallel Computation of Solutions to Convex
Feasibility Problems," Linear Algebra and Its Applications, Vol. 120, 1989, pp. 65-175.

doi:10.1016/0024-3795(89)90375-3

[7] Y. Censor and Z. Stavors, "Parallel Optimization: Theory, Algorithms, and Applications," Oxford University Press, Oxford, 1997.

[8] E. Candes, J. Romberg and T. Tao, "Robust Uncertainty Principles: Exact Signal Reconstruction from Highly Incomplete Frequency Information," IEEE Transactions on Information Theory, Vol. 52, No. 2, 2006, pp. 489-509. doi:10.1109/TIT.2005.862083

[9] E. Candes and M. Wakin, "An Introduction to Compressive Sampling," IEEE Signal Processing Magazine, Vol. 25, No. 2, 2008, pp. 21-30. doi:10.1109/MSP.2007.914731

[10] D. Donoho, “Compressed Sensing," IEEE Transactions on Information Theory, Vol. 52, No. 4, 2006, pp. 1289-1306. doi:10.1109/TIT.2006.871582

[11] C. E. Shannon, "Communication in the Presence of Noise," Proceedings of the IEEE, Vol. 86, No. 2, 1998, pp. 447-457. doi:10.1109/JPROC.1998.659497

[12] E. Candes and M. Wakin, "Enhancing Sparsity by Reweighted $L_{1}$ Minimization," Journal of Fourier Analysis and Applications, Vol. 14, No. 5-6, 2008, pp. 877-905. doi:10.1007/s00041-008-9045-X

[13] H. Yu and G. Wang, "Compressed Sensing Based Interior Tomography," Physics in Medicine and Biology, Vol. 54, No. 9, 2009, pp. 2791-2805. doi:10.1088/0031-9155/54/9/014

[14] X. Li and J. Zhu, "Convergence of Block Cyclic Projection and Cimmino Algorithms for Compressed Sensing Based Tomography," Journal of X-Ray Science and Technology, Vol. 18, No. 4, 2010, pp. 1-11.

[15] D. Butnariu, R. Davidi, G. T. Herman and I. G. Kazantsev, "Stable Convergence Behavior under Summable Perturbation of a Class Projection Methods for Convex Feasibility and Optimization Problems," IEEE Journal of Selected Topics in Signal Processing, Vo. 1, No. 4, 2007, pp. 540547.

[16] J. Zhu, X. Li, Y. Ye and G. Wang, "Analysis on the StripBased Projection for Discrete Tomography," Discrete Applied Mathematics, Vol. 156, No. 12, 2008, pp. 2359-2367. doi:10.1016/j.dam.2007.10.011

[17] X. Li and J. Zhu, "A Note of Reconstruction Algorithm of the Strip-Based Projection Model for Discrete Tomography," Journal of X-Ray Science and Technology, Vol. 16, No. 4, 2008, pp. 253-260.

[18] TEAM RADS. http://teamrads.com/ 